faire arriver $0 \mathrm{~cm}^{3} 5$ environ de réactif de Rupeau-Le-Mithouard (1). Le réactif traverse le chloroforme et donne, au contact du liquide aqueux ammoniacal, un anneau rouge caractéristique de la présence de l'acide picrique.

Nous avons observé qu'avec le filtrat débarrassé du lactose, la diazoréaction de Derrien est négative. Cela prouve que l'acide picrique est éliminé par le lait en nature, et non sous forme d'acide picramique, comme dans l'urine.

Toutefois, on peut, si on veut, effectuer la diazoréaction, à condition de réduire auparavant l'acide ṕicrique. Pour cela :

Dans une fiole conique, verser les $50 \mathrm{~cm}^{3}$ de filtrat restant. Ajouter $3 \mathrm{gr}$. de poudre de zinc, $6 \mathrm{~cm}^{3}$ de lessive de soude et chauffer pendant quelques minutes sur un bain-marie.

Après refroidissement et filtration, neutraliser exactement par l'acide sulfurique.

Prélever $20 \mathrm{~cm}^{3}$ de liquide, filtré si besoin, ajouter 11 gouttes de solution à $1 \%$ de nitrite de sodium et $X$ gouttes d'acide sulfurique dilué au quart; agiter. Ajouter ensuite XXX gouttes d'ammoniaque saturée de $\beta$-naphtol au moment du besoin; mélanger et agiter avec de l'éther. Ce dernier se sépare coloré en violet.

\title{
RECHERCHES SUR LE KÉFIR (2)
}

Le kéfir est une boisson d'origine caucasienne, préparée avec du lait de chèvre, de vache ou de brebis, fermenté sous l'influence d'un certain nombre de microorganismes qui coagulent finement la caséine et transforment le lactose en acide lactique, alcool et gaz carbonique. On obtient ainsi un liquide blanc, mousseux, de consistance plus ou moins crémeuse, d'odeur et de saveur agréables.

D'après SkLomowSKI, ]'origine du kéfir serait cette boisson connue sous le nom d' (ayran» préparée depuis fort longtemps par les montagnards des pentes-nord du Caucase. Ils obtenaient l'ayran en laissant séjourner pendant un certain temps le lait de leurs bêtes dans des outres réservées à cet usage, et qui n'étaient jamais lavées ni nettoyées ; jusqu'au jour où certains d'entre eux découvrirent dans ces outres des grains blanchâtres, disposés en grappes sur les

(1) Réactif :

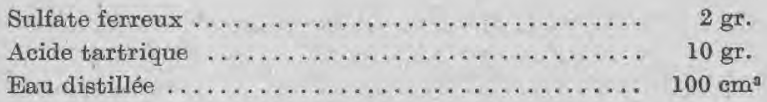

(2) Boris Bourounofw, Thèse de Doct. vét., Lyon, 1933. Travail du Laboratoire de Chimio de l'Ecole vétérinaire de Lyon. Analysé par R. Vurutume, ohef de travaux de chimie à 1'Ecole vétérinaire de Lyon. 
parois ; ces grains placés dans du lait frais, donnèrent une boisson meilleure que l'ayran lui-même, et qui fut appelée "kéfir ».

La préparation du kéfir fut gardée très longtemps secrète, car une légende voulait que ces grains eussent été envoyés sur terre par Mahomet à ses fidèles, avec interdiction de livrer le mode de préparation du kéfir aux dissidents, sous peine de voir les grains perdre leurs merveilleuses propriétés.

Depuis une cinquantaine d'années, cependant, cette boisson s'est répandue dans toute la Russie, puis dans d'autres pays; et dans certaines régions, sa préparation a même été industrialisée.

Dans son ouvrage, B. BounounOFF décrit d'abord les nombreux grains de kéfir vendus dans le commerce, puis aborde la délicate question de la bactériologie du kéfir; il nous indique ensuite les divers modes de préparation du kéfir, et termine par l'exposé de ses recherches sur la composition chimique et plus particulièrement sur la teneur en acide lactique et en alcool du kéfir.

\section{LES GRAINS DE KÉFIR}

Ce sont les éléments indispensables à la préparation du kéfir; ils renferment une flore microbienne complexe, fixée sur un support de caséine. Ces grains sont de deux sortes, suivant qu'ils sont :

a) frais ;

b) conservés par dessiccation.

Seuls les grains frais sont directement utilisables à la préparation du kéfir; on les plonge dans du lait, dans les conditions qui seront indiquées plus loin. A leur contact, le lait subit les modifications qui le transforment progressivement en kéfir, mais en même temps, les grains utilisés s'accroissent, et les diverses manipulations en détachent des fragments qui s'accroissent à leur tour; de sorte qu'à chaque préparation de kéfir correspond un accroissement du nombre des grains employés. Comme cet excès de grains ne trouve pas son application immédiate et que, d'autre part, les grains ne peuvent être conservés vivants qu'un temps relativement court, certains eurent l'idée d'augmenter leur temps de conservation en les desséchant au soleil.

C'est ainsi que l'on trouve dans le commerce, et surtout en Russie, des grains desséchés, qui se présentent sous forme de petites masses dures, irrégulières, de la grosseur moyenne d'une noisette, de coloration jaune ou brune. Les microorganismes y sont plongés dans une vie latente, solidement protégés par une gaine de caséine desséchée. On peut ainsi les conserver près d'un an.

Parfois aussi, ces grains secs sont pulvérisés, mélangés à du lactose et vendus soit sous forme de poudre, soit sous forme de comprimés (ferments kéfiriques du $D^{\mathrm{r}} L a$, du $D^{\mathrm{r}}$ Trainer; grains de kéfir $d u$ 
$D^{\mathrm{r}}$ Klebs, du $D^{\mathrm{r}}$ Timpe, etc.). Ces succédanés des grains naturels sont de qualité inférieure; leur conservation est moins longue et leur activité bien plus faible. Avant l'emploi, on doit préalablement procéder à la "revivification des grains desséchés".

On les laisse d'abord macérer pendant 5-8 heures dans de l'eau tiède fréquemment renouvelée, puis dans une solution de bicarbonate de soude à $10 \mathrm{gr}$. par litre; les grains se gonflent, deviennent élastiques et leur coloration s'éclaircit. Ils sont alors rincés, puis triés de façon à éliminer tous ceux qui sont trop mous, grisâtres ou translucides. Les autres sont alors placés dans dix fois leur poids de lait bouilli et refroidi à la température de 15 à $20^{\circ} \mathrm{C}$. Après 24 heures, les grains sont isolés, rincés à nouveau et réensemencés dans une quantité de lait bouilli et refroidi un peu plus forte que la veille. On recommence ainsi chaque jour.

En général, après 4 à 5 jours, le lait eommence à fermenter au niveau de ces grains, et certains d'entre eux, allégés par les bulles de $\mathrm{CO}^{2} \mathrm{qui}$ se dégagent à leur niveau, remontent à la surface.

$\mathrm{Au}$ bout de 7 à 10 jours en moyenne, tous les grains remontent à la surface quelques heures après l'ensemencement, et chacun d'eux est capable de coaguler en 24 heures trente à quarante fois son poids de lait.

A ce moment, la revivification est terminée et les grains, devenus complètement blanc mat, très élastiques, sont analogues aux grains frais, et peuvent être, comme eux, directement employés à la préparation du kéfir.

\section{BACTÉRIOLOGIE dU KÉFIR}

Le problème de la flore mierobienne des grains de kéfir est des plus difficile à résoudre; d'abord parce que les grains du commerce proviennent des sources les plus diverses, et surtout parce qu'il existe parmi eux de nombreux grains anormaux ou malades, renfermant les germes les plus variés : germes de la putréfaction, germes d'affections diverses (maladie de sécrétion de Podwissotzki), germes de fermentations parasites (fermentation acétique, butyrique...), etc. Aussi la question est-elle des plus controversée, chaque auteur ayant isolé une flore différente.

KERN, le premier, en 1881, montre qu'il existe dans les grains une symbiose entre une levure: Saccharomyces cerevisiae Meyen, à laquelle il attribue un rôle primordial, et des bactéries, dont la plus répandue, retrouvée en 1883 par KRANNHALS, se présente sous forme de bâtonnets, avec un renflement à chaque extrémité ; il la rapproche de B. subtilis et la nomme Dispora caucasica.

Vers 1900, BeIJERINK isole une autre levure: Saccharomyces kéfir, qui décompose le lactose en alcool et $\mathrm{CO}^{2}$, et un bacille : 
B. caucasicus, qu'il estime capable de coaguler finement la caséine, et dont l'optimum d'action est à $45^{\circ}$.

Fredonengich conteste la présence de la Dispora caucasica de Kern, qu'il considère comme parasite; il isole $B$. caucasicus, Saccharomyces kéfir, et, en outre, deux streptocoques. Pour cet auteur, B. caucasicus ne caille pas le lait, mais l'acidifie légèrement ; il n'a done qu'une action secondaire, l'action principale étant dévolue au Streptocoque $A$, qui coagule et acidifie le lait, et au Streptocoque $B$, qui hydrolyse le lactose et permet ainsi l'action de la levure.

En 1907, Mlle NIcolaIEWA isole B. caucasicus, qui provoque la fermentation lactique, et Torula kéfir, qui provoque la fermentation alcoolique du lactose. Cet auteur isole en outre de nombreux germes qa'il considère comme secondaires.

KUNTZE, en 1908, s'élève contre le rôle important attribué par la plupart des auteurs à $B$. caucasicus : il s'agit à son avis d'un hôte accidentel, mais non indispensable; d'ailleurs, il possède un optimum d'action élevé $\left(45^{\circ}\right)$, alors que le kéfir se prépare à la température optimum de $15-20^{\circ}$. Il isole plusieurs levures et plusieurs bacilles (Bacterium acidi lactici Leichmann, B. lactis aerogenes, ferments butyriques) et estime que les levures ont un rôle peu important, car il existe presque toujours des bacilles capables de provoquer la fermentation alcoolique; les levures seraient là pour favoriser et régulariser le processus.

Enfin, en 1924, Sanchez, Hallion et Lagnel isolent:

10 B. caucasicus (analogue au B. subtilis), qui acidifie légèrement le lait, coagule, solubilise et peptonise la caséine ;

$2^{\circ}$ Une levure, capable de fermenter directement le Jactosse, en le transformant en alcool et $\mathrm{CO}^{2}$ (done différente de la levure de Freudenreich);

$3^{\circ}$ Des ferments lactiques qui coagulent finement puis solubilisent partiellement la caséine, en même temps qu'ils acidifient le lait jusqu'à $100^{\circ}$ Dornic ;

$4^{\circ} \mathrm{De}$ nombreux autres germes secondaires.

De tout ceci, on doit actuellement se contenter de conclure qu'il existe dans les grains de kéfir une symbiose entre des levures et des bactéries, symbiose capable (comme le montre l'analyse du kéfir), d'une part de coaguler finement et de peptoniser en partie la caséine, d'autre part de transformer le lactose en acide lactique, alcool et $\mathrm{CO}^{2}$. Mais en l'état de nos connaissances, nous ne pouvons pas limiter avec certitude le rôle qui incombe à chacun des éléments constituants de cette symbiose.

\section{PRÉPARATION DU KÉFIR}

Tous les procédés de préparation du kéfir actuellement en usage 
dérivent de la méthode autrefois employée par les montagnards du Caucase. Ils plaçaient dans une outre quelques grains de kéfir et du lait; l'outre était alors fermée puis agitée de temps en temps. Au bout de 1 à 2 jours, suivant la température, le kéfir prêt à consommer était décanté, et les grains restés dans l'outre servaient à la confection d'un nouveau kéfir.

De nos jours, l'outre est remplacée par un récipient en verre ou émaillé. On emploie du lait de vache, de chèvre ou de brebis. Ce lait peut être entier ou écrémé, mais de toute manière, il y a avantage, pour éviter les fermentations parasites, à la stériliser par une ébullition préalable. Ce lait bouilli doit être ensuite ramené à la température ordinaire avant d'être mis au contact des grains de kéfir qui sont très sensibles à l'action de la chaleur.

On peut alors recourir à trois modes de préparation :

1. Préparation à partir des grains ;

2. Préparation à partir d'un levain mère;

3. Préparation à partir du kéfir lui-même.

1. Préparation par emploi immédiat des grains. - Les grains utilisés dans ce but sont frais, ou fraîchement revivifiés.

Dans un récipient, on place le lait bouilli et refroidi, et on lui ajoute les grains; la proportion de grains à employer est très variable suivant les régions : de 2 à $40 \mathrm{gr}$. par litre, soit en moyenne $20 \mathrm{gr}$. Le récipient est couvert d'un linge permettant l'aération, et le tout est disposé à l'obscurité, à une température de $15-20^{\circ}$. On l'agite toutes les 2-3 heures.

Au bout de 5 à 24 heures (ce temps est d'autant plus court que la quantité de grains est plus grande), on obtient le "kéfir jeune ». On sépare les grains par filtration sur un tamis et le liquide est mis dans des bouteilles. Ces bouteilles ne doivent être remplies qu'aux trois quarts, car la fermentation se poursuit, donne naissance à un dégagement de $\mathrm{CO}^{2}$ qui pourrait les faire éclater.

Ce kéfir doit être consommé dans les deux jours, ear ensuite le liquide devient trop acide; cependant, en plaçant les bouteilles dans une glacière à $0^{\circ}$, on peut conserver un bon kéfir près de huit jours ; il suffit d'agiter les flacons au moins une fois par jour.

2. Préparation à partir d'un levain mère. - C'est lo procédé russe par excellence; il consiste en une modification de la méthode précédente apportée dès 1870 par DIMITRIFFF, qui avait remarqué, à une époque où l'on ne possédait que de bien vagues notions sur les ferments, que la fermentation kéfirique une fois commencée, se prolongeait même après séparation du lait et des grains.

On commence d'abord par préparer le levain. Dans un récipient, on place des grains frais qu'on additionne de 40 à 50 fois leur poids 
de lait bouilli et refroidi à $15-20^{\circ}$. Le tout est placé à l'obscurité et remué toutes les 2 ou 3 heures.

Après 24 heures, les grains ont remonté à la surface, le levain est prêt; par filtration, on sépare un liquide de consistance crémeuse, d'odeur de levure et de saveur acide.

Pour obtenir le kéfir, le levain est mélangé à du lait bouilli refroidi : 1 partie de levain pour 4 parties de lait. Le mélange est distribué dans des bouteilles remplies aux trois quarts, qu'on conserve à $15-20^{\circ}$, en les agitant toutes les 2-3 heures.

Au bout de 1 jour, on obtient le "kéfir jeune ", liquide crémeux, de consistance homogène, très légèrement mousseux, de saveur douce et possédant l'arôme spécial du kéfir.

Après 2 jours, on a du "kéfir moyen ", c'est un liquide crémeux, mousseux, possédant un goût de crème acidifiée.

Vers le $3^{\mathrm{e}}$ jour, on obtient du "kéfir fort", très mousseux, parfois rejeté en partie avec les gaz à l'ouverture de la bouteille; ce kéfir est très aromatique, de saveur aigrelette et pique la langue.

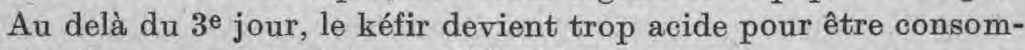
mable ; il est alors très riche en $\mathrm{CO}^{2}$, et la pression de ce gaz provoque parfois l'éclatement des flacons.

3. Préparation à partir du kéfir lui-même.-C'est le procédé le plus simple; il consiste à ajouter une certaine proportion de lait à du kéfir préparé par l'une des méthodes précédentes (en moyenne, 5 à 10 parties de lait pour 1 partie de kéfir).

$\mathrm{Au}$ bout de 24 heures, on obtient un nouveau kéfir, qui peut luimême être employé pour une nouvelle préparation ; et ainsi de suite. Mais le kéfir ainsi obtenu est de moins en moins " fort » et on ne peut guère dépasser une vingtaine de passages.

A côté de ces méthodes pratiques de préparation du kéfir, certains auteurs ont tenté de le préparer à l'aide de cultures pures des germes qu'ils avaient isolés des grains de kéfir, et cela dans le but de vérifier l'action qu'ils avaient attribuée à chacun des microorganismes. Il ne semble pas qu'ils aient obtenu des résultats concluants; signalons cependant KunTzE, d'une part, SANCHEz, HALLION et LAGNEL, d'autre part, qui prétendent avoir obtenu ainsi une boisson présentant tous les caractères d'un bon kéfir.

\section{COMPOSITION CHIMIQUE}

La composition chimique du kéfir a déjà été indiquée par Bogolubow, Veigmann. Elle subit évidemment des variations avec le mode de préparation, et surtout avec l'âge et la température (de préparation et de conservation). Ces variations intéressent particulièrement le taux du lactose (qui diminue), l'acidité et la quantité d'alcool (qui augmentent). 
Ce sont surtout les variations de ces trois éléments que B. BouRounofr a étudiées. L'acidité est évaluée en degrés Dornic ; le lactose est dosé par la méthode Bertrand, et l'alcool, par la méthode chronométrique de Nicloux après distillation d'environ un tiers du kéfir (tout l'alcool passe avec ce premier tiers).

Comme l'appréciation volumétrique est impraticable, par suite de la présence d'une quantité variable de bulles de $\mathrm{CO}^{2}$, toutes les déterminations précédentes sont faites en poids et rapportées à $1.033 \mathrm{gr}$. de kéfir (quantité moyenne de kéfir produite par un litre de lait).

B. BouRounofF étudie d'abord les variations de la composition chimique avec l'âge et la température, d'un kéfir préparé par la méthode russe.

Il part d'un lait présentant les caractères suivants :

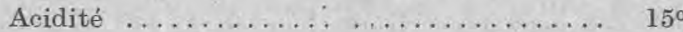

$$
\begin{aligned}
& \text { Lactose . . . . . . . . . . . . . . . } 49,96
\end{aligned}
$$

Ce lait est mélangé dans la proportion de 4 à 1 , avee un levain dont les caractères sont :

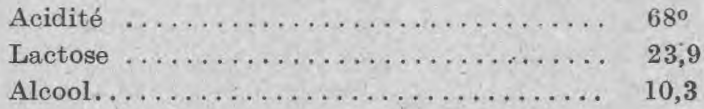

Le mélange, laissé à $17^{\circ}$, donne après 29 heures un kéfir jeune dont la composition est :

$\begin{array}{lll}\text { Acidité } & \ldots \ldots \ldots \ldots \ldots \ldots \ldots \ldots \ldots \ldots \ldots \ldots \ldots & 7^{72} \\ \text { Lactose } & \ldots \ldots \ldots \ldots \ldots \ldots \ldots \ldots \ldots \ldots \ldots \ldots & 31,6 \\ \text { Alcool } \ldots \ldots \ldots \ldots \ldots \ldots \ldots \ldots \ldots \ldots \ldots \ldots \ldots & 5,9\end{array}$

Ce kéfir est divisé en trois parties :

$$
\begin{aligned}
& N^{\circ} 1 \text {, conservé à } 17^{\circ} \text {; } \\
& \text { No }^{\circ} \text {, conservé à } 9^{\circ} \text {; } \\
& N^{\circ} 3 \text {, conservé à } 1^{\circ},
\end{aligned}
$$

\begin{tabular}{|c|c|c|c|}
\hline Age & Acidité & Lactose & Alcool \\
\hline 1 jour & $86^{\circ}$ & 25,4 & 8,3 \\
\hline 2 jours ................. & $94^{\circ}$ & 20,8 & 10,6 \\
\hline 3 jours .......... & $97^{\circ}$ & 14,1 & 13,4 \\
\hline 4 jours ........... & $98^{\circ} 5$ & 8,7 & 15,8 \\
\hline 5 jours $\ldots \ldots \ldots \ldots \ldots \ldots \ldots \ldots \ldots$ & $99^{\circ}$ & 5,7 & 17,4 \\
\hline
\end{tabular}

chacune d'elles étant analysée les jours suivants.

Voici les résultats :

Kéfir $n^{\circ} 1$ (conservé à $17^{\circ}$ ), 
Kéfir $n^{0} 2$ (conservé à $9^{\circ}$ ).

\begin{tabular}{|c|c|c|c|}
\hline Age & Acidité & Lactose & Alcool \\
\hline$\ldots \ldots \ldots \ldots \ldots \ldots \ldots \ldots \ldots$ & $78^{\circ}$ & 28,5 & 7,1 \\
\hline 2 jours $\ldots \ldots \ldots \ldots \ldots \ldots \ldots \ldots$ & $83^{\circ}$ & 26,2 & 7,9 \\
\hline 3 jours..$\ldots \ldots \ldots \ldots \ldots \ldots \ldots \ldots$ & $86^{\circ}$ & 24,6 & 8,7 \\
\hline 4 jours $\ldots \ldots \ldots \ldots \ldots \ldots \ldots \ldots$ & $87^{\circ}$ & 22,9 & 9,5 \\
\hline 5 jours $\quad \ldots \ldots \ldots \ldots \ldots \ldots \ldots \ldots$ & $88^{\circ}$ & 22,3 & 9,9 \\
\hline 6 jours & $88^{\circ}$ & 21,6 & 10,3 \\
\hline
\end{tabular}

Kéfir $n^{0} 3$ (conservé à $1^{\circ}$ ).

\begin{tabular}{|c|c|c|c|}
\hline Age & Acidité & Lactose & Alcool \\
\hline 5 & & & \\
\hline 1 jour & $73^{\circ}$ & 30,8 & 6,3 \\
\hline 6 jours $\ldots \ldots \ldots \ldots \ldots \ldots \ldots \ldots$ & $73^{\circ}$ & 29,2 & 7,1 \\
\hline 11 jours...................... & $73^{\circ}$ & 27,7 & 7,9 \\
\hline
\end{tabular}

Le $n^{\circ} 1$, conservé à une température normale, subit des variations rapides et n'est plus consommable au delà du 3 e jour; le $n^{\circ} 2$, conservé à $9^{\circ}$, est encore consommable le $6^{\mathrm{e}}$ jour (kéfir fort); enfin, le $n^{\circ} 3$, conservé à la glacière, subit de très faibles modifications, et constitue encore, le $11 \mathrm{e}$ jour, un kéfir moyen.

L'auteur termine par une observation relative à un kéfir préparé par passages successifs à partir d'un kéfir de 24 heures obtenu par la méthode russe. Chaque jour, il ensemence un lait de mélange du commerce (renfermant en moyenne $48 \mathrm{gr}$. de lactose) avec le kéfir préparé la veille, dans la proportion : 1 partie de kéfir pour 7 parties de lait (en poids). L'analyse est effectuée sur les kéfirs de deux jours ; ses résultats sont les suivants (à 170) :

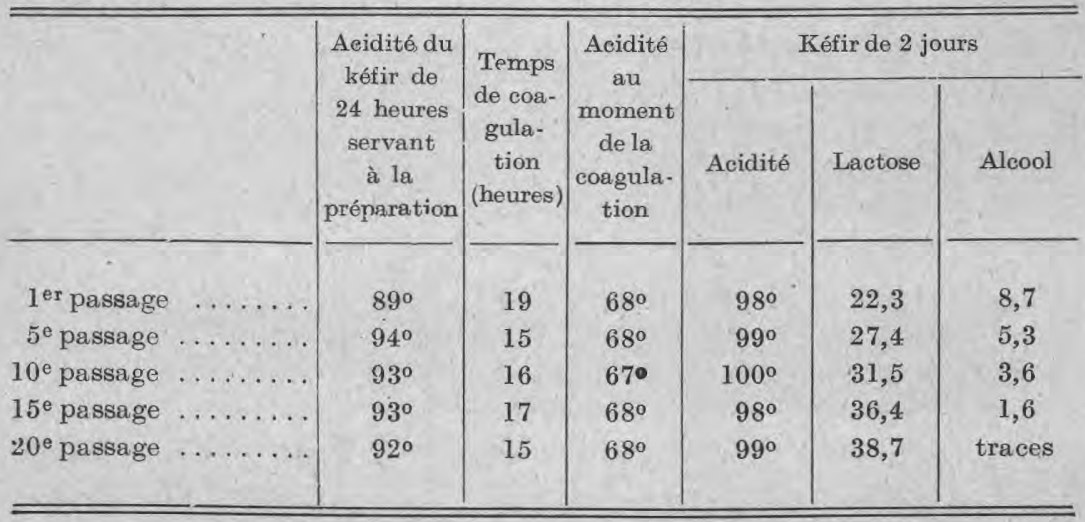


On voit que la fermentation kéfirique est pratiquement arrêtée au $20^{\circ}$ passage; aussi, B. Boणrounoff conclut-il qu'il s'agit là d'une méthode de préparation défectueuse, et que e'est au procédé russe qu'il faut donner la préférence.

\title{
REVUE
}

\section{LA PASTEURISATION DU LAIT PAR LE COURANT ÉLECTRIQUE}

\author{
par G. GÉNIN
}

Une des principales difficultés que l'on rencontre lorsqu'on procède à la pasteurisation du lait est due à des surchauffes locales qui se produisent dans certains points des appareils et qui communiquent au lait un gô̂t défectueux en diminuant par suite sa qualité. On a donc cherché à réaliser un chauffage du lait, c'est-à-dire une pasteurisation, au moyen du courant électrique, en faisant traverser le lait par ce courant. Les résultats ont été très satisfaisants et actuellement ce procédé, qui aux Etats-Unis est déjà appliqué dans de nombreuses installations, tend à s'étendre de plus en plus en Europe.

Il est possible de ehauffer le lait par courant électrique par suite de la présence dans ce lait de sels minéraux ou d'autres électrolytes en concentration suffisante. Il suffit de faire passer un courant alternatif dans le lait, l'amenée du courant se faisant par des électrodes de carbone et le lait constituant lui-même le milieu chauffant, ce qui évite toute surchauffe plus ou moins localisée.

Depuis que cette technique a été proposée à l'industrie laitière, des améliorations nombreuses ont été apportées à ce procédé, en particulier au cours de ces dernières années. Dans la pratique, une installation de pasteurisation par le courant électrique comprend les appareils suivants :

10 Un réservoir d'alimentation muni d'un flotteur qui règle une vanne et sert à régulariser la pression du liquide pénétrant dans l'appareil proprement dit. De ce réservoir, le lait est conduit dans des réchauffeurs-échangeurs où il est élevé à la température de $49^{\circ}$, la chaleur nécessaire à cette élévation de température étant fournie par le lait chaud sortant du pasteurisateur. A la sortie des réchauffeurs, le lait est pompé au moyen d'une pompe rotative dans un filtre, puis de là dans la chambre de pasteurisation. Cette chambre est constituée par une cuve rectangulaire portant des plaques de carbone sur ses côtés opposés. Ces plaques de carbone constituant les électrodes sont refroidies extérieurement par un courant d'eau froide et elles 